\title{
Neural stem cells induce M2 polarization of macrophages through the upregulation of interleukin-4
}

\author{
ZHUANGQI JI $^{1 *}$, XIANMING JIANG ${ }^{2 *},{\text { YUBIN } \mathrm{LI}^{3}, \mathrm{JIAN} \mathrm{SONG}^{4}, \mathrm{CUICUI} \mathrm{CHAI}^{4} \text { and XIAOFANG LU }}^{4}$ \\ ${ }^{1}$ Department of Gastrointestinal-Hepatobiliary Surgery, Shenzhen Longhua District Central Hospital, Shenzhen, \\ Guangdong 518110; ${ }^{2}$ Department of Ophthalmology, The Seventh Affiliated Hospital, Sun Yat-sen University, \\ Shenzhen, Guangdong 518107; ${ }^{3}$ The Reproductive Center, The First Affiliated Hospital, Sun Yat-sen University, \\ Guangzhou, Guangdong 510080; ${ }^{4}$ Department of Pathology, The Seventh Affiliated Hospital, Sun Yat-sen University, \\ Shenzhen, Guangdong 518107, P.R. China
}

Received March 30, 2020; Accepted July 31, 2020

DOI: $10.3892 /$ etm.2020.9277

\begin{abstract}
Macrophages are divided into two types: M1- and M2-type macrophages. Both types of macrophages serve important roles during the process of inflammation. M1-type macrophages release various pro-inflammatory cytokines, such as IL-1, IFN- $\gamma$ and other inflammatory mediators, such as nitric oxide, glutamate and reactive oxygen species to generate inflammation. In contrast, M2-type macrophages counteract the pro-inflammatory M1 conditions and promote tissue repair by secreting anti-inflammatory cytokines, such as IL-10. In spinal cord injury (SCI), an imbalance in M1/M2 macrophages leads to irreversible tissue destruction. Thus, it is crucial to increase the number of M2-type macrophages and promote M2 polarization of macrophages in SCI. Accordingly, in this study an in vitro co-culture system was established to investigate the effect of neural stem cells (NSCs) on macrophages. The results of the present study demonstrated that NSCs induced M2 polarization and suppressed M1 polarization of macrophages in an interleukin (IL)-4-dependent manner. Furthermore, the nuclear factor $(\mathrm{NF})-\kappa \mathrm{B} / \mathrm{p} 65$ signaling pathway was involved in the M1 polarization of macrophages and NSCs suppressed the activation of the NF- $\kappa \mathrm{B} / \mathrm{p} 65$ pathway in an IL-4-dependent manner to induce M2 macrophage polarization. These findings provide more insight into SCI and help to identify novel treatment strategies.
\end{abstract}

Correspondence to: Dr Xiaofang Lu, Department of Pathology, The Seventh Affiliated Hospital, Sun Yat-sen University, 628 Zhenyuan Road, Guangming, Shenzhen, Guangdong 518107, P.R. China

E-mail: zdluxiaofang2003@163.com

${ }^{*}$ Contributed equally

Key words: neural stem cell, macrophages, anti-inflammation, IL-4, p65

\section{Introduction}

Inflammatory processes are generally divided into four stages: Initiation, inflammation, resolution and tissue repair $(1,2)$. Macrophages mainly serve important roles throughout the whole process of inflammation and have different effects in different stages $(2,3)$. Consequently, macrophages have been divided into two types according to different and opposite functional states (pro-inflammatory and anti-inflammatory); these two types of macrophages are characterized by different phenotypes (1). A previous study suggested that macrophages differentiated into the M1 or M2 type are involved in the restoration of homeostasis and tissue repair by switching gene expression (3).

Macrophages are able to change their phenotype in response to numerous different stimuli and this process is termed polarization (4). In the 1990s, M2-type macrophages were first recognized as anti-inflammatory and tissue-repairing macrophages (5). This phenotype is different from that of typical inflammatory macrophages (M1) (5,6). M1-type macrophages are produced after monocytes are stimulated with lipopolysaccharide (LPS) or interferon- $\gamma$ (IFN- $\gamma$ ); M2-type macrophages are produced after monocytes are stimulated with interleukin (IL)-10 or IL-4 (1,7). In most wound repairs, M1-type macrophages are important in initiating the inflammatory response to produce exudation; in the later inflammatory stage, most of M1-type macrophages switch to the M2 phenotype to promote inflammation resolution and tissue repair (8). However, a previous study demonstrated that in spinal cord injury (SCI), the microenvironment of damaged spinal cord is consistently filled with M1-type macrophages that do not switch to the M2 phenotype (9). Ultimately, the consistent effect of M1-type macrophages results in irreversible spinal cord tissue destruction (9). Thus, it is important to promote M1 to $\mathrm{M} 2$ switching in macrophages and maintain the presence of M2-type macrophages to promote tissue repair in SCI.

Currently, regulating macrophage polarization using various agents has been considered a novel potential strategy for a number of diseases. For example, blocking CD64 with neutralizing antibodies induces M2 polarization of macrophages to decrease M1 cells and increase M2 cells in acute 
inflammatory diseases, such as ovarian cyst (10). In addition, some strategies involve cell-cell interactions. Cheng et al (11) demonstrated that conditioned medium from neural stem cells (NSCs) promoted M2 polarization of macrophages by suppressing the expression of inducible nitric oxide synthase (iNOS). Matsubara et al and Kano et al $(12,13)$ also reported that conditioned stem cell medium induced spinal cord tissue recovery that was associated with promoting M2 polarization of macrophages. Thus, it is considered that there is a specific interaction between NSCs and macrophages, but the mechanism is still unknown.

The present study explored the effect of NSCs on macrophages and demonstrated that NSCs induce M2 polarization and suppress M1-polarization of macrophages, which is beneficial for tissue repair and neural regeneration through the secretion of IL-4. Knockdown of IL-4 in NSCs by specific short hairpin (sh)RNA abolished the promotion of M2 polarization. NSCs suppressed activation of the NF- $\mathrm{kB} / \mathrm{p} 65$ pathway in an IL-4-dependent manner. Hence, the results of the present study demonstrated the novel effect of NSCs on macrophages and the underlying mechanism, which may provide useful information for its potential translational application in NSCs transplantation.

\section{Materials and methods}

NSC isolation and culturing. All procedures performed on experimental animals were approved by the Animal Care and Use Committee of Sun Yat-sen University (Guangdong, China).

Rats. All animal protocols were approved by China Council on Animal care and Sun Yet-San University Committee and have therefore been performed in accordance with the ethical standards laid down in the 1964 Declaration of Helsinki and its later amendments. Adult male Wistar rats (200-250 g; 6 weeks of age) were purchased from animal facility of Sun Yat-sen University and housed in stainless steel cages. Rats were fed under controlled environment at $25 \pm 3^{\circ} \mathrm{C}$, humidity of $40-65 \%$ and $12 \mathrm{~h} \mathrm{light/dark} \mathrm{cycle,} \mathrm{and} \mathrm{free} \mathrm{access} \mathrm{to} \mathrm{food}$ and drink water. Normal food (Animal Facility of Sun Yat-sen University) was provided.

Rats were anesthetized with $1 \%$ pentobarbital sodium $(40-45 \mathrm{mg} / \mathrm{kg})$ to cause minimum pain and sacrificed by $\mathrm{CO}_{2}$ asphyxiation. To be sacrificed, animals were placed into euthanasia cases, then $100 \% \mathrm{CO}_{2}$ were injected into cases at the filling rate of $30 \%$ per min. Death was confirmed by cardio-respiratory arrest and loss of reflexes. The duration of animal experiments was $<30 \mathrm{~min}$.

NSCs were isolated from the fetal brains of embryonic day 14 rats, which were extracted from pregnant Sprague-Dawley (SD) rats and identified as previously described (14). Briefly, the fetal brain tissue was mechanically dissected and dissociated in Hanks Balanced Salt Solution and the cell suspension was centrifuged at $4^{\circ} \mathrm{C}, 111 \mathrm{x}$ g for $5 \mathrm{~min}$. The supernatant was discarded and the cell pellet was diluted to a single-cell suspension. NSCs $\left(1 \times 10^{5}\right)$ were plated on a T25 culture flask (Corning Inc.) containing Dulbecco's modified Eagle's medium (DMEM)/F-12 nutrient mixture, 2\% B27, 1\% penicillin/streptomycin, 1\% l-glutamine (all purchased from
Gibco; Thermo Fisher Scientific, Inc.), $20 \mathrm{ng} / \mathrm{ml}$ fibroblast growth factor-2 (FGF-2) and $20 \mathrm{ng} / \mathrm{ml}$ epidermal growth factor (EGF; both purchased from PeproTech, Inc.). NSCs were cultured at $37^{\circ} \mathrm{C}$ in $5 \% \mathrm{CO}_{2}$ and were passaged weekly by digestion with Accutase (EMD Millipore) in the medium described above. All NSCs (passage 2-3) used in this study were between passages 2 and 4 .

To induce neural differentiation, cells were plated at a density of $2 \times 10^{5}$ cells/well in 6 - or 12 -well tissue-culture plates and allowed to adhere for $24 \mathrm{~h}$ at $37^{\circ} \mathrm{C}$, at which time cells were switched to neural differentiated medium consisting of basic medium supplemented with $2 \%$ B27, $1 \%$ penicillin/streptomycin and $1 \%$ l-glutamine. The medium was changed every 2-3 days $(14,15)$.

Monocytes were isolated from the femurs of adult SD rats as previously described (2). In co-culture system, cells were differentiated into the macrophages when culturing in DMEM supplemented with $20 \mathrm{ng} / \mathrm{ml}$ macrophage colony-stimulating factor (M-CSF) (PeproTech, Inc.) at $37^{\circ} \mathrm{C}$ in $5 \% \mathrm{CO}_{2}$ for 7 days (12).

Reverse transcription-quantitative (RT-q)PCR. Total RNA was extracted from cells according to the manufacturer's protocol (16) and $2 \mu \mathrm{g}$ of total DNA-free RNA was used to synthesize cDNA using the ReverTra Ace ${ }^{\circledR}$ qPCR RT kit (Toyobo Life Science). The reactions were set up in 96-well plates using $1 \mu \mathrm{l}$ cDNA with Thunderbird SYBR qPCR Mix (Toyobo Life Science), to which gene-specific forward and reverse PCR primers were added. RT-qPCR was performed under the following conditions: $95^{\circ} \mathrm{C}$ for $10 \mathrm{~min}$, followed by 40 cycles of $95^{\circ} \mathrm{C}$ for $10 \mathrm{sec}$ and $55^{\circ} \mathrm{C}$ for $34 \mathrm{sec}$. These analyses were performed to detect the expression of CD58, iNOS, CD206, CD163, IL-4, IL-10, IL-13, tumor necrosis factor (TNF)- $\alpha$, IFN- $\gamma$ and $\beta$-actin was used as an internal control. Primer sequences were as demonstrated in Table I.

Western blotting analysis. Cells were lysed in RIPA buffer (25 mM Tris-HCl pH 7.5, $150 \mathrm{mM} \mathrm{NaCl}, 1 \% \mathrm{Na}$-deoxycholale, 0.5 M EDTA, 1\% Nonidet P40, 0.1\% SDS, Beijing Solarbio Science \& Technology Co., Ltd; cat. no. R0010), total protein was extracted, and the protein concentration was determined with a bicinchoninic acid assay. SDS-PAGE gels (10 and $12 \%$ ) were loaded with $20 \mu \mathrm{g} /$ lane of total protein. The separated proteins were transferred by electro blotting to PVDF membranes. The membranes were blocked with $5 \%$ non-fat dry milk in TBST at room temperature for $2 \mathrm{~h}$ (50 mM Tris; pH 7.6; $150 \mathrm{mM} \mathrm{NaCl}$; and $0.1 \%$ Tween-20) and incubated with the primary antibody overnight at $4^{\circ} \mathrm{C}$ in 5\% non-fat dry milk in TBST. Immunology-labeling was detected using ECL reagent (Invitrogen; Thermo Fisher Scientific, Inc.). The primary antibodies used were as follows: Anti-p65 (1:1,000, Room temperature, 45 min, Cell Signaling Technology, Inc; cat. no. 8242), anti-phospho-p65 (1:1,000; Room temperature, $45 \mathrm{~min}$; Cell Signaling Technology Inc; cat. no. 93H1), anti-IL-4 antibody (1:500; Room temperature, 45 min, Proteintech Group, Inc; cat. no. 66142), anti- $\beta$-actin (1:10,000; Room temperature, 45 min, Sigma-Aldrich; Merck KGaA; cat. no. A5316) and anti-GAPDH (1:10,000; Room temperature, 45 min, Sigma-Aldrich; Merck KGaA; cat. no. G9545). The secondary antibody (1:1,000; Cell 
Table I. Primer sequences used for reverse transcription-quantitative PCR.

\begin{tabular}{ll}
\hline Gene & \multicolumn{1}{c}{ Sequence (5'-3') } \\
\hline CD85 & F: TCACGGCTGAGATTCGACAG \\
& R: GTTTTGTGACGGACTGAGGTTAT \\
Inducible nitric & F: GAGGAGAGAGATCCGGTTCACA \\
oxide synthase & R: CCGCATTAGCACAGAAGCAA \\
CD206 & F: GACAGATATGAACAAGCATTCC \\
& R: TGAACATCTGAGAGTCCTGTC \\
CD163 & F: CGTCACCTTGGAAACAGAGACAG \\
& R: AGATCTCCACACGTCCAGAACAG \\
IL-4 & F: ACCTTGCTGTCACCCTGTTCT \\
IL-10 & R: AGCTCGTTCTCCGTGGTGT \\
& F: CAGACCCACATGCTCCGAGA \\
IL-13 & R: CAAGGCTTGGCAACCCAAGTA \\
& F: GCTCTCGCTTCGCTTGGTGGTC \\
Tumor & R: CATCCGAGGCCTTTTGGTTACAG \\
necrosis factor- $\alpha$ & R: GTTGTCTTTGAGATCCATGCCATT \\
Interferon- $\gamma$ & F: TCGCACCTGATCACTAACTTCTTC \\
$\beta$-actin & R: CGACTCCTTTTCCGCTTCC \\
& F: CCTCTTTGCATGTCTCACTC \\
& R: AATGTCACGCACGATTCC
\end{tabular}

IL, interleukin; F, forward; R, reverse.

Signaling Technology, Inc.) were incubated for $1 \mathrm{~h}$ at room temperature.

Transfection. Recombinant lentiviruses for IL-4 silencing (shIL-4) and control lentivirus (scramble) were obtained from Shanghai Genechem Co., Ltd. For transfection, cells in the log phase were plated at a concentration of $1 \times 10^{5}$ cells/well in 6-well plates and transfected with the scramble and shIL-4 (MOI: 20) in DMEM/F12 with 10\% FBS. Polybrene (Beijing Solarbio Science \& Technology Co., Ltd; cat. no. H8761) was added as an enhancing reagent to improve transduction efficiency at a concentration of $10 \mathrm{mg} / \mathrm{ml}$. After $8 \mathrm{~h}$, the medium was changed with fresh complete medium. Cells were harvested for the co-culture system $24 \mathrm{~h}$ after transfection.

Immunofluorescence confocal microscopy. Macrophages (5x103/well) were transferred to flat-bottom 96-well plates at the indicated density. After being treated and incubated, the cells were fixed with $4 \%$ paraformaldehyde in $15 \mathrm{~min}$ at room temperature, permeabilized with $0.5 \%$ Triton X-100 in PBS for $10 \mathrm{~min}$ at room temperature, blocked with PBS containing $1 \% \mathrm{BSA}$ in $15 \mathrm{~min}$ at room temperature, and then incubated with an antibody against p65 (1:50; Cell Signaling Technology Inc.) overnight at the $4^{\circ} \mathrm{C}$. After being washed with PBS, the cells were incubated with an Alexa Fluor-488-conjugated anti-rabbit secondary antibody (1:1,000; Invitrogen; Thermo Fisher Scientific, Inc; cat. no. A11029.) for $1 \mathrm{~h}$ at room temperature before being imaged with a confocal microscope using a LCPlanFl objective (magnification x200; Olympus Corporation).
Enzyme linked immunosorbent assay for the quantitation of $I L-4, I L-10$ and IL-13. The ELISA of IL-4 (Abcam; cat. no. ab215089), IL-10 (Abcam; cat. no. ab185986) and IL-13 (Abcam; cat. no. ab100553) were performed using ELISA kits. The protocol was as follows: Prepare all reagents, working standards, and the culture medium. Remove unused microplate strips from the plate frame, return them to the foil pouch containing the desiccant pack, and reseal. Wash each well three times with PBS (300 $\mu 1 /$ well) using a squirt bottle, multi-channel pipette, manifold dispenser or autowasher. Complete removal of liquid at each step is essential to good performance. Remove any remaining Wash Buffer by aspirating or decanting. Invert the plate and blot it against clean paper towels. Add $100 \mu \mathrm{l}$ of each serially diluted protein standard or culture medium per well including a zero standard. Ensure reagent addition is uninterrupted and completed within $15 \mathrm{~min}$. Cover/seal the plate and incubate for $2 \mathrm{~h}$ at room temperature. Add $100 \mu \mathrm{l}$ of Detection Antibody in working concentration to each well. Cover/seal the plate and incubate for $1 \mathrm{~h}$ at room temperature. Add $200 \mu \mathrm{l}$ of Substrate Solution to each well. Incubate for $20 \mathrm{~min}$ at room temperature. Protect from light. Add $50 \mu \mathrm{l}$ of Stop Solution to each well. If color change does not appear uniform, gently tap the plate to ensure thorough mixing. Determine the optical density of each well within $20 \mathrm{~min}$, using a microplate reader set to $450 \mathrm{~nm}$.

Flow cytometric analysis. Single-cell suspensions were prepared and stained with fluorochrome-conjugated antibodies (Invitrogen; Thermo Fisher Scientific, Inc, cat. no. A35029) at the room temperature for $1 \mathrm{~h}$. Data were collected on a BD LSRII flow cytometer (BD Biosciences) and analyzed with FlowJo software V2.0 (Tree Star, Inc.). Data were acquired as the fraction of labeled cells within a live-cell gate set for 50,000 events. For flow cytometric sorting, cells were stained with specific antibodies (APC Mouse Anti-Human HLA-DR; 1:200; BD Biosciences; cat. no. 559868; PE Mouse Anti-Human CD163; 1:200; BD Biosciences; cat. no. 556018; BV421 Mouse Anti-Human CD206, 1:200, BD Biosciences; cat. no. 564062) and isolated on a BD FACSAria cell sorter (BD Biosciences) (17).

Statistical analysis. Data were represented as the mean \pm SD of three independent repeats. Shapiro-Wilk test was used to analyze the normality of data and one-way ANOVA with Levene's test was used to test the homogeneity of variance, followed by the Bonferroni post hoc test for multiple comparisons. The analyses were performed using SPSS (version 16.0; SPSS, Inc.). $\mathrm{P}<0.05$ was considered to indicate a statistically significant difference.

\section{Results}

NSCs induce M2-polarization and suppress M1-polarization of macrophages. To determine the interactions between NSCs and macrophages, an in vitro co-culture system was established to investigate the effect of NSCs on macrophages. The frequencies of macrophages by flow cytometry was measured. The results demonstrated that the M1-type macrophages $\left(\mathrm{HLA}-\mathrm{DR}^{+}\right)$were significantly reduced from $0.18 \pm 0.03$ to $0.02 \pm 0.01 \%$ after co-culture with NSCs (Fig. 1A and B). The 
RT-qPCR results demonstrated that the mRNA expression levels of the M1-type macrophage markers (CD85 and iNOS) were significantly decreased in co-cultured cells compared with M0-macrophages alone (Fig. 1C). In contrast, flow cytometric analysis demonstrated that the M2-type macrophages $\left(\mathrm{CD}^{2} 06^{+}\right.$and $\left.\mathrm{CD} 163^{+}\right)$were significantly increased from $1.87 \pm 0.17$ to $32.93 \pm 3.43 \%$ after co-culture with NSCs (Fig. 1D and E). Additionally, the RT-qPCR results demonstrated that the mRNA expression levels of the M2-type macrophage markers (CD206 and CD163) were significantly increased in co-cultured cells compared with M0-type macrophages cultured alone (Fig. 1F). These results suggested that NSCs induce M2 polarization and suppress M1 polarization in macrophages.

NSCs induce M2 polarization of macrophages in an IL-4-dependent manner. Previous studies have demonstrated that stimulation with different cytokines affects macrophage polarization (M1 or M2) $(4,18,19)$. IL-4 or IL-13 stimulation induces M2 polarization; TNF- $\alpha$ or IFN- $\gamma$ stimulation induces M1 polarization (1). To confirm whether these cytokines were involved in the interactions between NSCs and macrophages, NSCs were cultured in neural differentiation medium. The RT-qPCR and ELISA results demonstrated that the mRNA and protein expression levels of IL-4, but not IL-13 or IL-10 were significantly increased in cells with the increase in time (Fig. 2A and B). In contrast, no significant differences were observed in the mRNA or protein expression levels of TNF- $\alpha$ and IFN- $\gamma$ during neural differentiation (Fig. 2C and D). To further confirm whether the secretion of IL-4 was crucial for NSC-induced M2 polarization, a target-specific lentivirus was used to knock down the expression levels of IL-4 in NSCs $\left(\mathrm{NSC}^{\text {shIL-4}}\right)$, then the NSC ${ }^{\text {shIL-4 }}$ were co-cultured with macrophages. The RT-qPCR results demonstrated that the mRNA expression levels of the M1-type macrophage marker (CD85 and iNOS) were significantly increased in macrophages co-cultured with NSCs ${ }^{\text {shIL-4 }}$ compared with those of macrophages cultured alone (Fig. 2E). By contrast, the mRNA expression levels of the M2-type macrophages marker (CD206 and CD163) were significantly decreased in macrophages co-cultured with $\mathrm{NSC}^{\text {shIL-4 }}$ compared with those of macrophages cultured alone (Fig. 2F). These results suggested that NSCs induce M2 polarization of macrophages in an IL-4-dependent manner.

NSCs suppress the nuclear factor $(N F)-\kappa B / p 65$ pathway to promote M2-polarization of macrophages in an IL-4-dependent manner. Activation of the $\mathrm{NF}-\kappa \mathrm{B} / \mathrm{p} 65$ pathway by inflammatory cytokines such as TNF- $\alpha$ in macrophages induce the M1 polarization of macrophages $(20,21)$. To determine whether IL-4 was crucial for NSC-mediated inhibition of NF-KB/p65 pathway activation, a target-specific lentivirus was used to knock down the expression levels of IL-4 in NSCs (Fig. 3A), then the NSC ${ }^{\text {shIL-4 }}$ were co-cultured with macrophages. The results demonstrated that silencing IL-4 expression significantly diminished the inhibitory effect of NSCs on p65 translocation (from cytoplasm to nucleus) and p65 phosphorylation in macrophages (Fig. 3B and C). These results suggested that NSCs suppress the NF- $\kappa B / p 65$ pathway to promote M2 polarization of macrophages in an IL-4-dependent manner.

\section{Discussion}

The present study established an in vitro co-cultured system to investigate the interactions between NSCs and macrophages and the effect of NSCs on the polarization of macrophages. The results demonstrated that M1-type macrophages markers, such as CD85 and iNOS, were significantly decreased in macrophages that were co-cultured with NSCs compared with those of macrophages cultured alone. In addition, the number of HLA-DR-positive cells was decreased in this co-cultured system. By contrast, M2-type macrophage markers, such as CD206 and CD163, were significantly increased in macrophages that were co-cultured with NSCs compared with those of macrophages cultured alone. In addition, CD206 and CD163 double-positive cells were decreased in this co-cultured system. Additionally, IL-4 mRNA and protein expression levels were significantly increased during neural differentiation. Knockdown of IL-4 expression in NSCs diminished the M2 polarization-promoting effect on macrophages and the $\mathrm{NF}-\mathrm{\kappa B} / \mathrm{p} 65$ pathway was the primary pathway involved in this process.

Macrophages are important in a number of processes, such as inflammation, tumorigenesis and tissue repair (22). M1/M2 polarization is a specific characteristic of macrophages that divides macrophages into M1-type macrophages and M2-type macrophages (23). M1-type macrophages are termed inflammatory macrophages because this type of cell is related to inflammatory production; M2-type macrophages are termed healing macrophages because they have an anti-inflammatory effect and have been reported to promote tissue repair (1).

As previously mentioned, a number of diseases are caused by the imbalances in M1/M2 macrophage phenotypes $(24,25)$. For example, M1-type macrophages attack tumors and prevent tumor growth (26). In contrast, infiltration of M2-type macrophages promotes tumor deterioration (27). In chronic inflammatory processes, inflammatory cytokine production by M1-type macrophages leads to cancer or dysregulation and promote tissue destruction, followed by the M2-type macrophage response, promoting tissue repair in these situations (28). In SCI, macrophages also have an important role in the pathological mechanism. Previous studies reported that both types of macrophages, which have opposing neurotoxic and neuroprotective functions, are recruited $(8,29,30)$. It has been reported that consistent infiltration of M1-type macrophages is strongly related to numerous pathological processes of SCI, such as neuronal death and demyelination $(31,32)$. On the other hand, studies have reported that the M2-type macrophages switches from the M1-type macrophages. M2-type macrophages act as anti-inflammatory cells, expressing IL-4 and IL-10 and generating TGF- $\beta$, while downregulating the expression of numerous proinflammatory cytokines, such as IL-1, IL-6, IL-18, TNF- $\alpha$ and IFN $\gamma$ (8). These mediators of M2-type macrophages suppress inflammatory responses and promote tissue remodeling and repair $(33,34)$. Thus, increasing the population of M2-type macrophages and maintaining the survival of this type of cell in the microenvironment of damaged tissue may represent a promising strategy for tissue repair after $\mathrm{SCI}$.

A number of clinical trials have reported the efficacy of the stem cell transplantation as a potential strategy to repair 

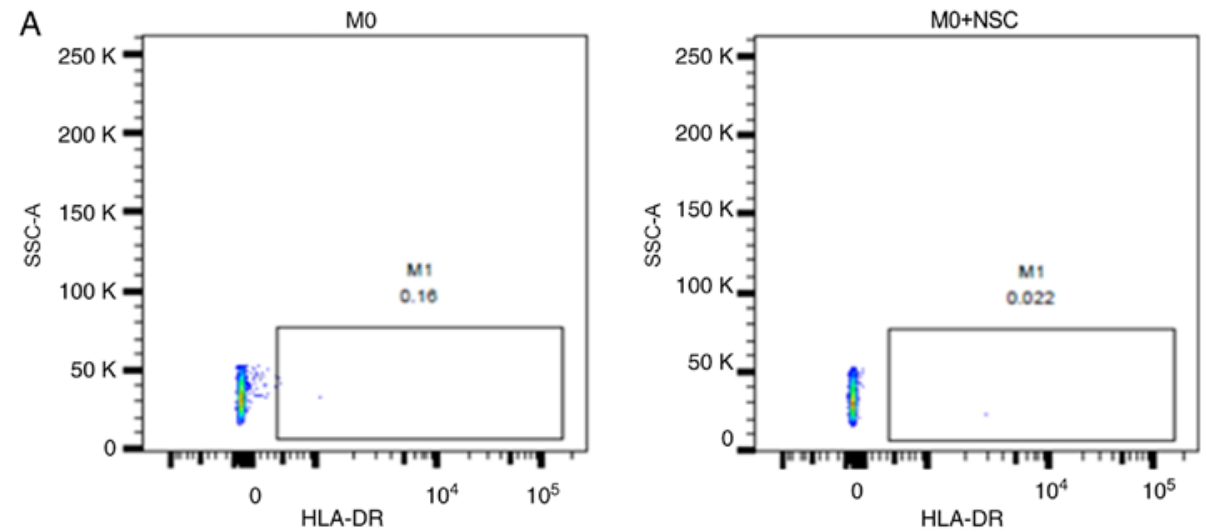

B

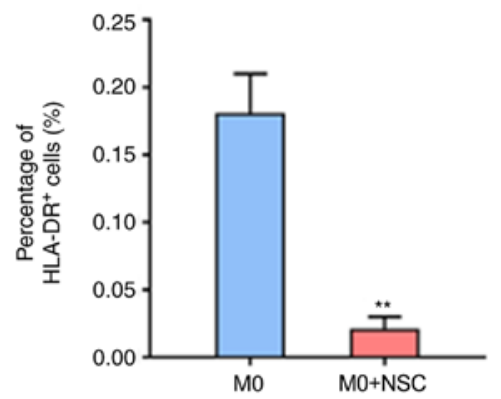

C

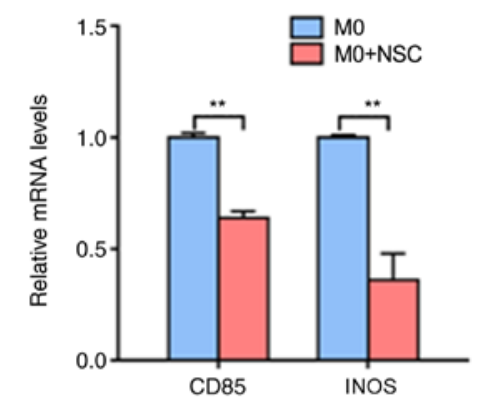

D
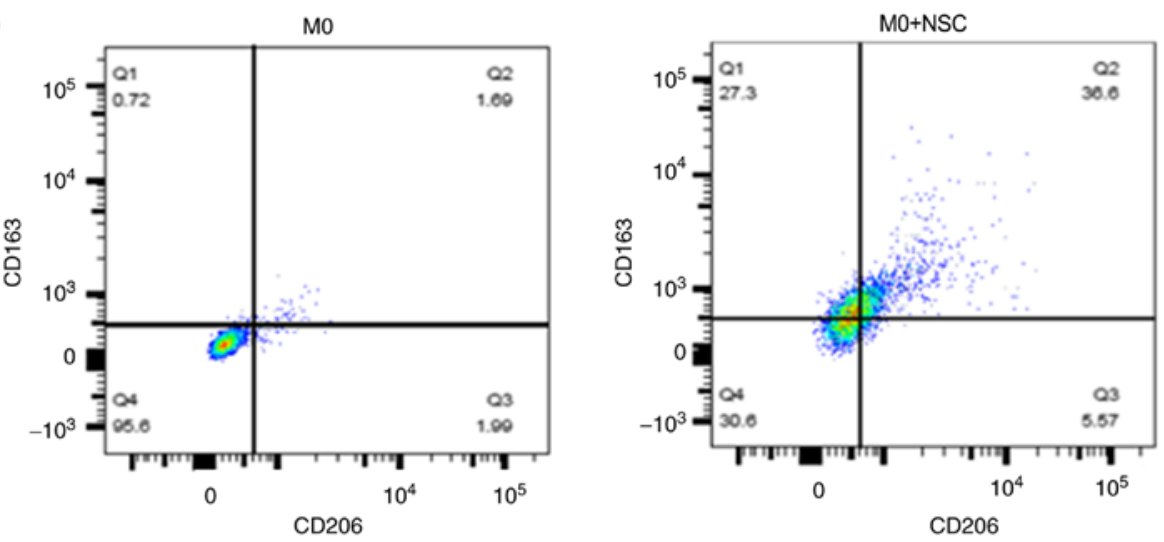

$E$

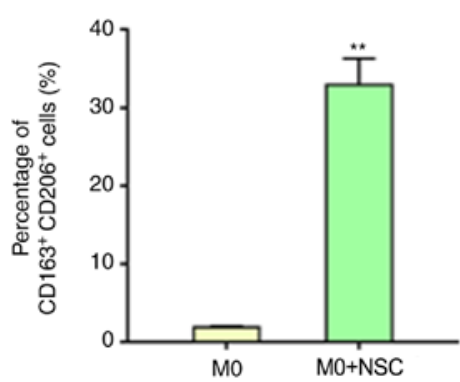

$\mathrm{F}$

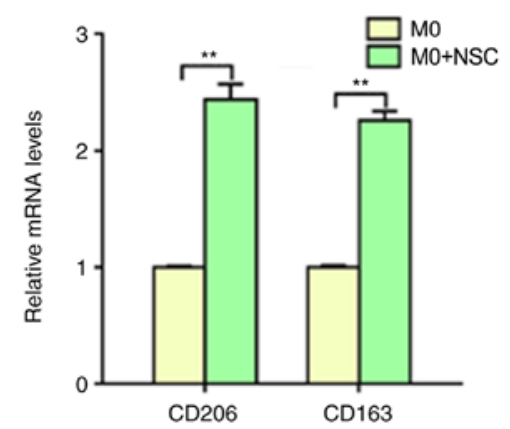

Figure 1. NSCs induce M2-polarization and suppress M1-polarization of macrophages. (A) Flow cytometric analyses of M1-type macrophages (HLA-DR $)$ in macrophages co-cultured with NSCs. (B) Quantification of the macrophage number for part A. (C) RT-qPCR analyses of CD85 and inducible nitric oxide synthase expression in macrophages cocultured with NSCs. (D) Flow cytometric analyses of M2-type macrophages $\left(\mathrm{CD} 206^{+} \mathrm{CD} 163^{+}\right)$in macrophages co-cultured with NSCs. (E) Quantification of the macrophage number for part D. (F) RT-qPCR analyses of CD206 and CD163 expression in macrophages cocultured with NSCs. ${ }^{* *} \mathrm{P}<0.01$. NSCs, neural stem cells; RT-qPCR, reverse transcription-quantitative PCR; iNOS, inducible nitric oxide synthase; M0, non-polarized macrophage.

damaged tissue and recover locomotor functional after SCI $(15,35,36)$. These studies demonstrated that the transplanted cells differentiate into neurons, which mainly rebuilt neural circuits to promote tissue repair and locomotor function recovery (37). In addition, the current study indicated that transplanted cells have been considered to modulate the polarization of macrophages to improve the microenvironment. However, the mechanism by which transplanted NSCs affect 
A

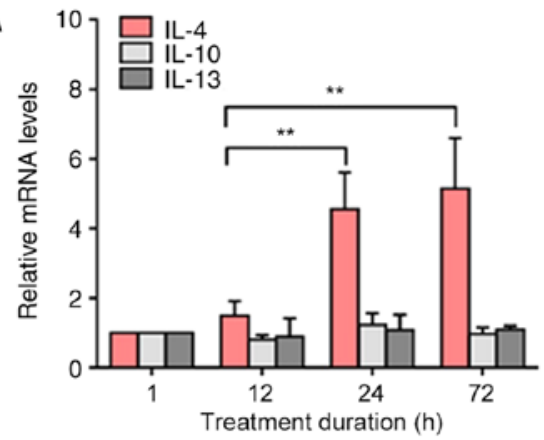

C

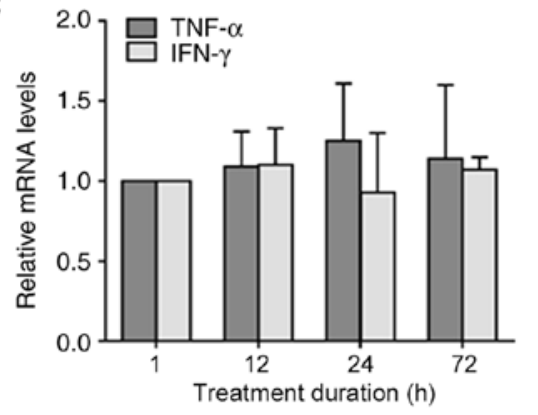

E

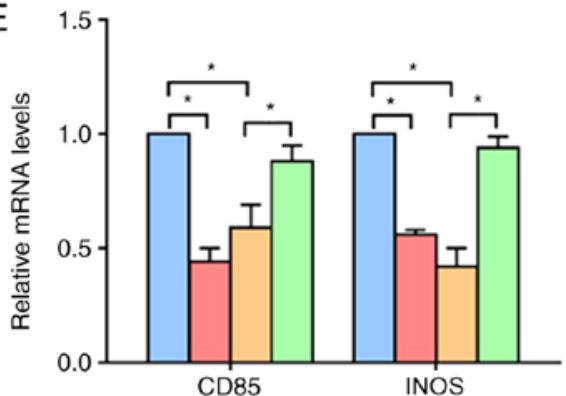

B

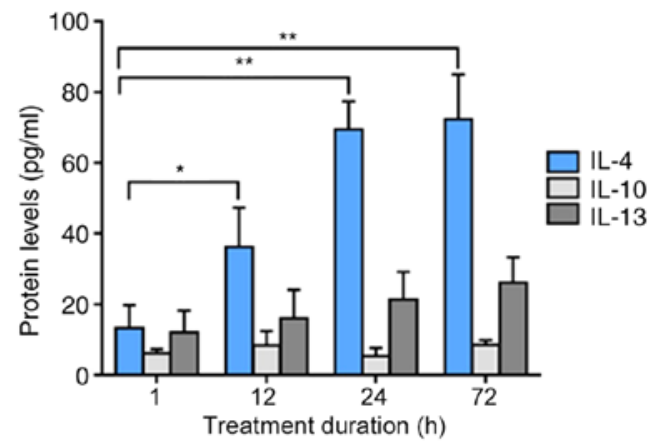

D

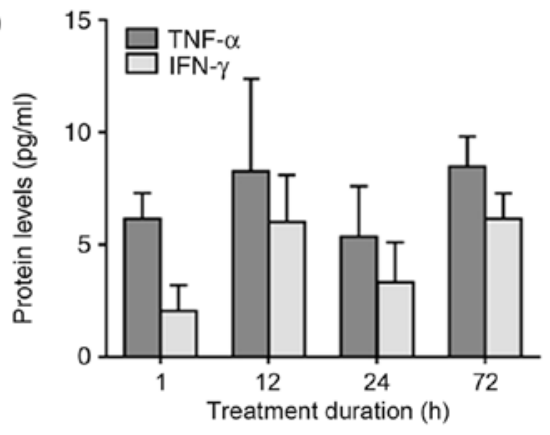

F

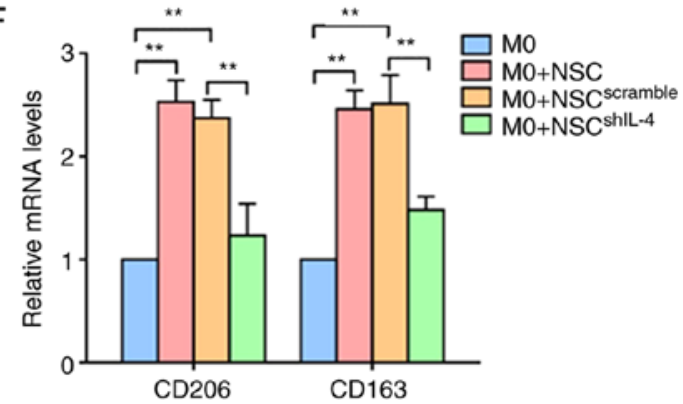

Figure 2. NSCs induce M2 polarization of macrophages by upregulating IL-4. (A) RT-qPCR analyses of IL-4, IL-10 and IL-13 expression in NSCs during neural differentiation. (B) ELISA analyses of IL-4, IL-10 and IL-13 concentrations in the culture medium of NSCs during neural differentiation. (C) RT-qPCR analyses of TNF- $\alpha$ and IFN- $\gamma$ expression in NSCs during neural differentiation. (D) ELISA analyses of TNF- $\alpha$ and IFN- $\gamma$ concentrations in the culture medium of NSCs during neural differentiation. (E and F) RT-qPCR analyses of CD85, inducible nitric oxide synthase, CD206 and CD163 expression in macrophages co-cultured with NSCs. " $\mathrm{P}<0.05,{ }^{* *} \mathrm{P}<0.01$. NSCs, neural stem cells; IL, interleukin; RT-qPCR, reverse transcription-quantitative PCR; TNF, tumor necrosis factor; IFN, interferon; INOS, inducible nitric oxide synthase; M0, non-polarized macrophage.

macrophages in the inflammatory microenvironment is still unclear. A previous study reported that the medium from NSCs has anti-inflammatory effects in vitro and in vivo, suppressing the expression of inflammatory cytokines in macrophages and injured spinal cord tissues (11). Matsubara et al (12) reported that conditioned medium from stem cells induced spinal cord tissue recovery by promoting M2 polarization of macrophages. To the best of our knowledge, the current study, provided the first evidence of the interaction between NSCs and macrophages. The results of the present study demonstrated that NSCs promoted M2 polarization in macrophages in an in vitro co-cultured system. Nakajima et al (38) demonstrated similar results that MSC transplantation promoted M2 polarization of macrophages and prevented M1 polarization of macrophages through the secretion of IL-4 and IL-13.

The underlying mechanism of NSC-induced M2 polarization of macrophages remains unclear. M1-type macrophages are produced after monocytes are stimulated with LPS or IFN- $\gamma$, and M2-type macrophages are produced after monocytes are stimulated with IL-10 or IL4 (7). Based on these findings, this study investigated whether IL-4,
IL-10 and IL-13 are involved in the interactions between NSCs and macrophages. The results of the present study demonstrated that the production of IL-4, but not IL-10 or IL-13, was increased in NSCs during neural differentiation. Additionally, knockdown of IL-4 expression in NSCs diminished the promotion of M2 polarization in the co-culture system. These findings suggested that NSCs induce M2 polarization of macrophages in an IL-4-dependent manner, which has not been reported previously to the best of our knowledge.

M1-type macrophages have strong cytotoxicity and anti-proliferative activities. They have been reported to promote neuron death, prevent neuronal differentiation and tissue destruction after SCI (39-41). Activation of the NF- $\kappa \mathrm{B} / \mathrm{p} 65$ pathway by inflammatory cytokines such as TNF- $\alpha$ and IL-1 in macrophages is important for M1 polarization $(42,43)$. The present study investigated the effect of NSCs on the $\mathrm{NF}-\kappa \mathrm{B} / \mathrm{p} 65$ pathway in macrophages; the results demonstrated that NSCs suppress the activation of $N F-\kappa B / p 65$ signaling by suppressing the phosphorylation and translocation of p65 in an IL-4-dependent manner. Taken together, the results of the 
A

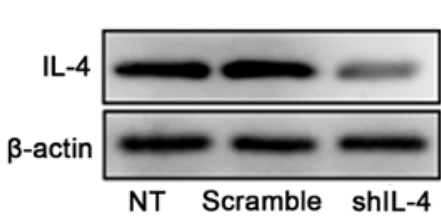

B

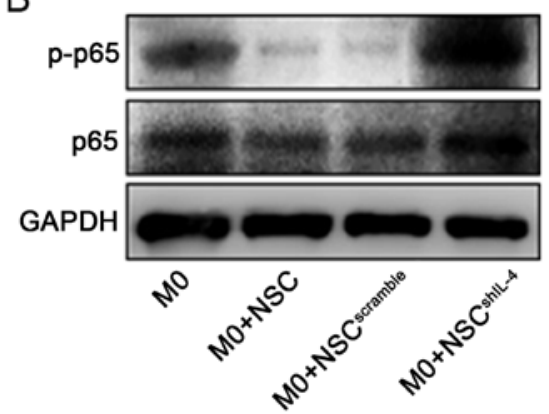

C



Figure 3. NSCs induce M2 polarization of macrophages by suppressing the nuclear factor- $\kappa$ B/p65 pathway in an IL-4-dependent manner. (A) Western blot analyses of IL-4 expression in NSCs transfected with scramble and short hairpin IL-4. (B) Western blot analyses of p65 phosphorylation in macrophages co-cultured with NSCs. (C) Immunofluorescence staining demonstrating the nuclear localization of p65 in macrophages co-cultured with NSCs. Scale bar, $50 \mu \mathrm{m}$. NSCs, neural stem cells; IL, interleukin; sh, short hairpin; p-p65, phosphorylated p65; M0, non-polarized macrophage.

present study suggested that NSCs induce M2 polarization and suppress the activation of $\mathrm{NF}-\kappa \mathrm{B} / \mathrm{p} 65$ signaling to prevent $\mathrm{M} 1$ polarization in an IL-4-dependent manner. In future studies, the effect and mechanism of M2-type macrophage protection of neurons and promotion of neuronal differentiation in SCI will be investigated.

There are some limitations of the current study; in vivo experiments are better to indicate the effects of IL-4 on improved tissue repair in SCI. Additionally, the current study should discuss more information regarding the mechanisms of IL-4 induced macrophage polarization. Also, studies on the negative effects of M1 macrophage on the neuronal differentiation are required in the future.

In conclusion, the results of the present study suggested that NSCs secrete the cytokine IL-4 to induce M2 polarization of macrophages and suppress activation of $N F-\kappa B / p 65$ signaling to prevent M1 polarization of macrophages during neural differentiation. The results of the present study may provide useful information to overcome the challenges of tissue repair in SCI and propose a potential therapeutic strategy for improving locomotor function recovery.

\section{Acknowledgement}

The authors would like to thank Dr Yue Hu (The First Affiliated Hospital of Sun Yat-sen University) for their assistance with the experiments.

\section{Funding}

This study was supported by the National Natural Science Foundation of China (grant no. 81971151) and the Guangdong Natural Science Foundation (grant no. 2019A1515010241; 2020A1515010265).

\section{Availability of data and materials}

The datasets used and/or analyzed during the present study are available from the corresponding author on reasonable request.

\section{Authors' contributions}

ZJ and XL designed the study. ZJ, XJ and XL conducted the study. YL, JS and CC collected the data. ZJ, XJ, JS, CC, and XL analyzed the data. ZJ, XJ, YL, and XL interpreted the data. ZJ, XJ and XL drafted the manuscript. ZJ and XJ revised the manuscript. All authors take responsibility for the integrity of the data analysis. All authors read and approved the final manuscript.

\section{Ethics approval and consent to participate}

This study was approved by the Animal Care and Use Committee of Sun Yat-sen University (approval no. 4557, Guangzhou, China). 


\section{Patient consent for publication}

Not applicable.

\section{Competing interests}

The authors declare that they have no competing interests.

\section{References}

1. Funes SC, Rios M, Escobar-Vera J and Kalergis AM: Implications of macrophage polarization in autoimmunity. Immunology 154 : 186-195, 2018.

2. Murray PJ and Wynn TA: Protective and pathogenic functions of macrophage subsets. Nat Rev Immunol 11: 723-737, 2011.

3. Varga T, Mounier R, Horvath A, Cuvellier S, Dumont F, Poliska S, Ardjoune H, Juban G, Nagy L and Chazaud B: Highly dynamic transcriptional signature of distinct macrophage subsets during sterile inflammation, resolution, and tissue repair. J Immunol 196: 4771-4782, 2016.

4. Mosser DM and Edwards JP: Exploring the full spectrum of macrophage activation. Nat Rev Immunol 8: 958-969, 2008.

5. Stein M, Keshav S, Harris N and Gordon S: Interleukin 4 potently enhances murine macrophage mannose receptor activity: A marker of alternative immunologic macrophage activation. J Exp Med 176: 287-292, 1992.

6. Fahlman C, Jacobsen FW, Veiby OP, McNiece IK, Blomhoff HK and Jacobsen SE: Tumor necrosis factor-alpha (TNF-alpha) potently enhances in vitro macrophage production from primitive murine hematopoietic progenitor cells in combination with stem cell factor and interleukin-7: Novel stimulatory role of p55 TNF receptors. Blood 84: 1528-1533, 1994.

7. He Y, Gao Y, Zhang Q, Zhou G, Cao F and Yao S: IL-4 switches microglia/macrophage M1/M2 polarization and alleviates neurological damage by modulating the JAK1/STAT6 pathway following ICH. Neuroscience 437: 161-171, 2020.

8. Kong X and Gao J: Macrophage polarization: A key event in the secondary phase of acute spinal cord injury. J Cell Mol Med 21: 941-954, 2017.

9. Kigerl KA, Gensel JC, Ankeny DP, Alexander JK, Donnelly DJ and Popovich PG: Identification of two distinct macrophage subsets with divergent effects causing either neurotoxicity or regeneration in the injured mouse spinal cord. J Neurosci 29: 13435-13444, 2009.

10. Akinrinmade OA, Chetty S, Daramola AK, Islam MU, Thepen T and Barth S: CD64: An attractive immunotherapeutic target for M1-type macrophage mediated chronic inflammatory diseases. Biomedicines 5: 56, 2017.

11. Cheng Z, Bosco DB, Sun L, Chen X, Xu Y, Tai W, Didier R, Li J, Fan J, He X and Ren Y: Neural stem cell-conditioned medium suppresses inflammation and promotes spinal cord injury recovery. Cell Transplant 26: 469-482, 2017.

12. Matsubara K, Matsushita Y, Sakai K, Kano F, Kondo M, Noda M, Hashimoto N, Imagama S, Ishiguro N, Suzumura A, et al: Secreted ectodomain of sialic acid-binding Ig-like lectin-9 and monocyte chemoattractant protein-1 promote recovery after rat spinal cord injury by altering macrophage polarity. J Neurosci 35: 2452-2464, 2015.

13. Kano F, Matsubara K, Ueda M, Hibi $\mathrm{H}$ and Yamamoto A: Secreted ectodomain of sialic acid-binding ig-like lectin-9 and monocyte chemoattractant protein-1 synergistically regenerate transected rat peripheral nerves by altering macrophage polarity. Stem Cells 35: 641-653, 2017.

14. Chen N, Cen JS, Wang J, Qin G, Long L, Wang L, Wei F, Xiang Q, Deng DY and Wan Y: Targeted inhibition of leucine-rich repeat and immunoglobulin domain-containing protein 1 in transplanted neural stem cells promotes neuronal differentiation and functional recovery in rats subjected to spinal cord injury. Crit Care Med 44: e146-e157, 2016.

15. Li X, Peng Z, Long L, Tuo Y, Wang L, Zhao X, Le W and Wan Y: Wnt4-Modified NSC transplantation promotes functional recovery after spinal cord injury. FASEB J 34: 82-94, 2020.

16. Livak KJ and Schmittgen TD: Analysis of relative gene expression data using real-time quantitative PCR and the 2(-Delta Delta C(T)) method. Methods 25: 402-408, 2001.
17. Yang Q, Shi M, Shen Y, Cao Y, Zuo S, Zuo C, Zhang H, Gabrilovich DI, Yu Y and Zhou J: COX-1-Derived thromboxane A2 plays an essential role in early B-cell development via regulation of JAK/STAT5 signaling in mouse. Blood 124: 1610-1621, 2014.

18. Orecchioni M, Ghosheh Y, Pramod AB and Ley K: Macrophage polarization: Different gene signatures in M1(LPS $\left.{ }^{+}\right)$vs. classically and M2(LPS') vs. alternatively activated macrophages (vol 10, 1084, 2019). Front Immunol 24: 1084, 2020.

19. Miller JE, Ahn SH, Marks RM, Monsanto SP, Fazleabas AT, Koti M and Tayade C: IL-17A Modulates peritoneal macrophage recruitment and $\mathrm{m} 2$ polarization in endometriosis. Front Immunol 14: 108. 2020.

20. Gu X, Zhang Y,Li D, Cai H, Cai L and Xu Q: N6-Methyladenosine demethylase FTO promotes M1 and M2 macrophage activation. Cell Signal 69: 109553, 2020.

21. Zhang L, Zhang J, Jiang X, Yang L, Zhang Q, Wang B, Cui L and Wang X: Hydroxytyrosol inhibits LPS-induced neuroinflammatory responses via suppression of TLR-4-mediated NF-kappaB P65 activation and ERK signaling pathway. Neuroscience 426: 189-200, 2020.

22. Bagheri M, Mostafavinia A, Abdollahifar MA, Amini A, Ghoreishi SK, Chien S, Hamblin MR, Bayat S and Bayat M: Combined effects of metformin and photobiomodulation improve the proliferation phase of wound healing in type 2 diabetic rats. Biomed Pharmacother 123: 109776, 2020.

23. Milich LM, Ryan CB and Lee JK: The origin, fate, and contribution of macrophages to spinal cord injury pathology. Acta Neuropathol 137: 785-797, 2019.

24. Liu YC, Zou XB, Chai YF and Yao YM: Macrophage polarization in inflammatory diseases. Int J Biol Sci 10: 520-529, 2014.

25. Labonte AC, Tosello-Trampont AC and Hahn YS: The role of macrophage polarization in infectious and inflammatory diseases. Mol Cells 37: 275-285, 2014.

26. Bai YH, Yin KM, Su T, Ji F and Zhang S: CTHRC1 in ovarian cancer promotes M2-like polarization of tumor-associated macrophages via regulation of the STAT6 signaling pathway. Onco Targets Ther 13: 5743-5753, 2020.

27. Gabrilovich DI, Ostrand-Rosenberg S and Bronte V: Coordinated regulation of myeloid cells by tumours. Nat Rev Immunol 12: 253-268, 2012.

28. Dvorak HF: Tumors: Wounds that do not heal-redux. Cancer Immunol Res 3: 1-11, 2015.

29. Hauk TG, Muller A, Lee J, Schwendener R and Fischer D: Neuroprotective and axon growth promoting effects of intraocular inflammation do not depend on oncomodulin or the presence of large numbers of activated macrophages. Exp Neurol 209: 469-482, 2008

30. Li SY, Nie K, Zhang QX, Guo ML, Qiu YH, Li Y, Gao Y and Wang L: Macrophage migration inhibitory factor mediates neuroprotective effects by regulating inflammation, apoptosis and autophagy in Parkinson's disease. Neuroscience 416: 50-62, 2019.

31. Ghasemlou N, Lopez-Vales R, Lachance C, Thuraisingam T, Gaestel M, Radzioch D, and David S: Mitogen-Activated protein kinase-activated protein kinase 2 (MK2) contributes to secondary damage after spinal cord injury. J Neurosci 30: 13750-13759, 2010.

32. Ghasemlou N, Bouhy D, Yang J, López-Vales R, Haber M, Thuraisingam T, He G, Radzioch D, Ding A and David S: Beneficial effects of secretory leukocyte protease inhibitor after spinal cord injury. Brain 133: 126-138, 2010.

33. Mantovani A, Biswas SK, Galdiero MR, Sica A and Locati M: Macrophage plasticity and polarization in tissue repair and remodelling. J Pathol 229: 176-185, 2013.

34. Marchetti V, Yanes O, Aguilar E, Wang M, Friedlander D, Moreno S, Storm K, Zhan M, Naccache S, Nemerow G, et al: Differential macrophage polarization promotes tissue remodeling and repair in a model of ischemic retinopathy. Sci Rep 76: 1038, 2011.

35. Ahuja CS, Nori S, Tetreault L, Wilson J, Kwon B, Harrop J, Choi D and Fehlings MG: Traumatic spinal cord injury-repair and regeneration. Neurosurgery 80: S9-S22, 2017.

36. Saberi H, Firouzi M, Habibi Z, Moshayedi P, Aghayan HR, Arjmand B, Hosseini K, Razavi HE and Yekaninejad MS: Safety of intramedullary schwann cell transplantation for postrehabilitation spinal cord injuries: 2-Year follow-up of 33 cases. J Neurosurg Spine 15: 515-525, 2011.

37. Assinck P, Duncan GJ, Hilton BJ, Plemel JR and Tetzlaff W: Cell transplantation therapy for spinal cord injury. Nat Neurosci 20: 637-647, 2017. 
38. Nakajima H, Uchida K, Guerrero AR, Watanabe S, Sugita D, Takeura N, Yoshida A, Long G, Wright KT, Johnson WE and Baba H: Transplantation of mesenchymal stem cells promotes an alternative pathway of macrophage activation and functional recovery after spinal cord injury. J Neurotrauma 29: 1614-1625, 2012.

39. Kaushal V, Koeberle PD, Wang Y and Schlichter LC: The $\mathrm{Ca}^{2+}$-activated $\mathrm{K}^{+}$channel $\mathrm{KCNN} 4 / \mathrm{KCa} 3.1$ contributes to microglia activation and nitric oxide-dependent neurodegeneration. J Neurosci 27: 234-244, 2007.

40. Bao F, Bailey CS, Gurr KR, Bailey SI, Rosas-Arellano MP, Dekaban GA and Weaver LC: Increased oxidative activity in human blood neutrophils and monocytes after spinal cord injury. Exp Neurol 215: 308-316, 2009.
41. Rathore KI, Kerr BJ, Redensek A, Lopez-Vales R, Jeong SY, Ponka P and David S: Ceruloplasmin protects injured spinal cord from iron-mediated oxidative damage. J Neurosci 28 : 12736-12747, 2008.

42. Kroner A, Greenhalgh AD, Zarruk JG, Passos Dos Santos R, Gaestel $M$ and David S: TNF and increased intracellular iron alter macrophage polarization to a detrimental M1 phenotype in the injured spinal cord. Neuron 83: 1098-1116, 2014

43. Brykczynska U, Geigges M, Wiedemann SJ, Dror E, Boni-Schnetzler M, Hess C, Donath MY and Paro R: Distinct transcriptional responses across tissue-resident macrophages to short-term and long-term metabolic challenge. Cell Rep 30: $1627-1643,2020$ 